\title{
Parametric 2-metric spaces and some fixed point results
}

\author{
Vildan Çetkin
}

Department of Mathematics, Kocaeli University, Kocaeli, Turkey

Received: 18 July 2019, Accepted: 18 December 2019

Published online: 27 December 2019.

\begin{abstract}
The main goal of the present article is to describe the notion of a parametric 2-metric space which is a nonlinear generalization of the parametric metric, and consider some fixed point theorems in the parametric 2-metric spaces. We first propose the elementary definitions and the notions of parametric 2-metric spaces. Then, we present and prove some fixed point theorems in a complete parametric 2-metric spaces.
\end{abstract}

Keywords: Parametric metric, 2-metric, convergence, fixed point theorem.

\section{Introduction}

Fixed point theorems are fundamental tools to prove the existence and uniqueness of the solutions to various mathematical models such as integral equations, partial differential equations and variational inequalities and so on. In the last decades different forms of metric spaces such as b-metric spaces [2], D-metric spaces [5], S-metric spaces [18], G-metric spaces [14], cone metric spaces [7] and so on, have been defined and some fixed point theorems have been observed by many authors, in these spaces. One of these generalizations is so called 2-metric was introduced by Gahler [6] as follows:

Definition 1. [6] Let $X$ be a non-empty set and $\rho$ be a mapping from $X \times X \times X$ to $\mathbb{R}^{+}=[0, \infty)$, that is $\rho: X \times X \times X \rightarrow \mathbb{R}^{+}$ satisfying the following conditions:

(1) for every pair of distinct points $x, y$ there exists a point $z \in X$ such that $\rho(x, y, z) \neq 0$.

(2) $\rho(x, y, z)=0$ if and only if at least two of the three points are same.

(3) $\rho(x, y, z)=\rho(x, z, y)=\rho(y, z, x)$, for all $x, y, z \in X$.

(4) $\rho(x, y, z) \leq \rho(x, y, w)+\rho(x, w, z)+\rho(w, y, z)$, for all $x, y, z, w \in X$.

Then $\rho$ is called a 2-metric on $X$ and the pair $(X, \rho)$ is called a 2-metric space. One can think of $\rho(x, y, z)$ as measuring how far are the points $x, y, z$ from being "aligned" or "colinear". The prototypical example of a 2-metric space is obtained by setting $\rho(x, y, z)$ equal to the area of the triangle spanned by $x, y, z$.

Notice that in the above generalizations, only a 2-metric space is not topologically equivalent to an ordinary metric. For instance, every metric space is first countable, but this is not true in general, for 2-metric spaces [13]. Then there was no easy relationship between results obtained in 2-metric spaces and metric spaces. In particular, the fixed point theorems on 2-metric spaces and metric spaces may be unrelated easily. For the details on 2-metric spaces and fixed point theorems on 2-metric spaces, the readers may refer to $[1,4,10,13,15]$.

The notion of a parametric metric space as one of the generalization of the metric space, has been defined by Hussain et al. [8] as follows: 
Definition 2. [8] Let $X$ be a non-empty set and $P: X \times X \times(0, \infty) \rightarrow \mathbb{R}^{+}$be a mapping. Then the mapping $P$ is called a parametric metric if it satisfies the following conditions:

(P1) $P(x, y, t)=0$ for all $t>0$ if and only if $x=y$.

(P2) $P(x, y, t)=P(y, x, t)$, for all $x, y \in X$ and all $t>0$.

(P3) $P(x, y, t) \leq P(x, z, t)+P(z, y, t)$, for all $x, y, z \in X$ and all $t>0$.

Then the pair $(X, P)$ is called a parametric metric space.

Nowadays the authors still studying on fixed point and common fixed point theorems of parametric metric spaces and its generalized versions such as parametric S-metric, parametric b-metric, parametric $N_{b}$-metric (see $\left.[3,9,12,16,17,19,20]\right)$.

In the present study, besides the above linear generalizations of parametric metric notions, we aim to make contribution to this area by giving the nonlinear generalization; the notion of a parametric 2-metric. As a result, we present this new notion. Also, in order to clarify the idea, we describe fundamental definitions and we observe some topological properties. Then, we consider different fixed point and common fixed point results in these spaces.

\section{Parametric 2-metric spaces}

In this section, we define the notion of a parametric 2-metric and propose some elementary concepts such as an open ball, convergent sequences, Cauchy sequences, continuity and etc.

Definition 3. Let $X$ be a non-empty set and $d: X \times X \times X \times(0, \infty) \rightarrow \mathbb{R}^{+}$be a mapping which satisfies the following conditions

(M1) For every $x, y \in X$ with $x \neq y$ there exists $z \in X$ such that $d(x, y, z, t) \neq 0$ for all $t>0$

(M2) $d(x, y, z, t)=0$, for all $t>0$, if at least two of $x, y, z \in X$ are the same

(M3) $d(x, y, z, t)=d(z, y, x, t)=d(z, x, y, t)=d(y, x, z, t)=d(x, z, y, t)=d(y, z, x, t)$ for all $x, y, z \in X$ and for all $t>0$

(M4) $d(x, y, z, t)=d(x, y, w, t)+d(x, w, z, t)+d(w, y, z, t)$, for all $x, y, z, w \in X$ and all $t>0$.

Then $d$ is called a parametric 2-metric on $X$ and the pair $(X, d)$ is called a parametric 2-metric space, which will be sometimes denoted by $X$ if there is no confusion. One can think of $d(x, y, z, t)$ as measuring how far are the points $x, y, z$ from being "aligned" or "colinear" according to parameters $t>0$.

Example 1. Let $X=\mathbb{R}$ be the set of real numbers and $f:(0, \infty) \rightarrow(0, \infty)$ be a continuous function. Then the mappings $d_{1}, d_{2}: \mathbb{R}^{3} \times(0, \infty) \rightarrow \mathbb{R}^{+}$defined as follows are parametric 2-metrics on $X$,

$d_{1}(x, y, z, t)=f(t)|(x-y)(x-z)(y-z)|$ and $d_{2}(x, y, z, t)=f(t)(|x-y|+|y-z|+|x-z|)$, for every $x, y, z \in X$ and for all $t>0$.

Example 2. Let $(X, M, N, *, \diamond)$ be a triangular intuitionistic fuzzy 2-metric space. Define the mapping $d: X \times X \times X \times$ $(0, \infty) \rightarrow \mathbb{R}^{+}$by $d(x, y, z, t)=\frac{1}{M(x, y, z, t)-1}$ for all $x, y, z \in X$ and for all $t>0$. Then $d$ is a parametric 2-metric on $X$.

Example 3. Let $X=f \mid f:(0, \infty) \rightarrow \mathbb{R}$ is a function $\}$ and the mapping $d: X^{3} \times(0, \infty) \rightarrow \mathbb{R}^{+}$be defined by $d(f, g, h, t)=$ $|f(t)-g(t)|+|g(t)-h(t)|$ for each $f, g, h \in X$ and for each $t>0$. Then the pair $(X, d)$ is a parametric 2-metric space.

Lemma 1. Let $(X, P)$ be a parametric metric space and the mapping $d: X^{3} \times(0, \infty) \rightarrow \mathbb{R}^{+}$be defined by $d(x, y, z, t)=$ $P(x, y, t)+P(y, z, t)+P(x, z, t)$, for all $x, y, z \in X$ and for all $t>0$. Then $d$ is a parametric 2-metric on $X$. This implies that, each parametric metric induces a parametric 2-metric on the same universal set.

Proof. It is clear to verify from Definition 1 and Definition 2. 
Definition 4. Let $(X, d)$ be a parametric 2-metric space and $x, y \in X, r \geq 0$. Then the set defined by

$$
B(x, y, r)=\{z \in X \mid d(x, y, z, t)<r, \text { for all } t>0\}
$$

is called an open ball in $X$, centered at $x$ and $y$ with radius $r$.

The topology generated by the collection of all open balls as a subbasis is called a parametric 2-metric topology on $X$, and it is denoted by $\tau$. Members of $\tau$ are called 2-open sets and their complements, 2-closed sets.

Lemma 2. Let $(X, d)$ be a parametric 2-metric space and $G \subseteq X$. Then $G$ is a 2-open set if and only iffor every $x \in G$ there are finite numbers of points $y_{1}, y_{2}, \ldots, y_{n} \in X, r_{1}, r_{2}, \ldots, r_{n}>0$ such that $x \in B\left(x, y_{1}, r_{1}\right) \cap B\left(x, y_{2}, r_{2}\right) \cap \cdots \cap B\left(x, y_{n}, r_{n}\right) \subseteq$ G.

Proof. The sufficiency of the claim is evident from the fact that the intersection of the open balls

$$
B\left(x, y_{1}, r_{1}\right) \cap B\left(x, y_{2}, r_{2}\right) \cap \cdots \cap B\left(x, y_{n}, r_{n}\right)
$$

is 2-open. So, it is sufficient to prove the converse. Let $G \subseteq X$ be a 2-open set and $x \in G$. Then there exists a finite number of open balls $B\left(x_{i}, y_{i}, r_{i}\right), i=1,2, \ldots, m$ such that

$$
x \in B\left(x_{1}, y_{1}, r_{1}\right) \cap B\left(x_{2}, y_{2}, r_{2}\right) \cap \cdots \cap B\left(x_{n}, y_{n}, r_{n}\right) \subseteq G .
$$

Since $x \in B\left(x_{i}, y_{i}, r_{i}\right)$, then $d\left(x, x_{i}, y_{i}, t\right)=s_{i}(t)<r_{i}$, for all $i \in\{1,2, \ldots, m\}$ and for all $t>0$. Choose $\varepsilon_{i}(t)<\frac{r_{i}-s_{i}(t)}{2}$ for all $t>0$. In this case, we may conclude that $B\left(x, x_{i}, \varepsilon_{i}(t)\right) \cap B\left(x, y_{i}, \varepsilon_{i}(t)\right) \subseteq B\left(x_{i}, y_{i}, r_{i}\right)$, for all $i \in\{1,2, \ldots, m\}$ and all $t>0$. Therefore, for any $t>0$, we have

$$
x \in \bigcap_{i=1}^{m}\left(B\left(x, x_{i}, \varepsilon_{i}(t)\right) \cap B\left(x, y_{i}, \varepsilon_{i}(t)\right)\right) \subseteq \bigcap_{i=1}^{m} B\left(x_{i}, y_{i} r_{i}\right) \subseteq G
$$

This completes the proof.

Proposition 1. Each parametric 2-metric topological space $(X, \tau)$ is a $T_{1}$-space.

Proof. Let $x, y \in X \quad(x \neq y)$ be two points. Then by (M1) of Definition 2, there exists $z \in X$ such that $d(x, y, z, t)>0$, for all $t>0$. Say $r(t):=\frac{d(x, y, z, t)}{2}>0$, for all $t>0$. Fix some $t_{1}>0$, say $r_{1}=r\left(t_{1}\right)$. Then, the balls $B\left(x, z, r_{1}\right)$ and $B\left(y, z, r_{1}\right)$ are 2-open sets with $x \in B\left(x, z, r_{1}\right), y \notin B\left(x, z, r_{1}\right)$ and $x \notin B\left(y, z, r_{1}\right), y \in B\left(y, z, r_{1}\right)$. This completes the proof.

Definition 5. Let $(X, d)$ be a parametric 2-metric space and $\left(x_{n}\right)_{n \in \mathbb{N}}$ be a sequence in $X$. Then

(1) $\left(x_{n}\right)$ is said to be convergent to $x \in X$ written as $x_{n} \rightarrow x$, when $n \rightarrow \infty$ or $\lim _{n \rightarrow \infty} x_{n}=x$, if for every $a \in X$ and for all $t>0, \lim _{n \rightarrow \infty} d\left(x_{n}, x, a, t\right)=0$.

(2) $\left(x_{n}\right)$ is said to be Cauchy in $X$ if for every $a \in X$ and for all $t>0, \lim _{n, m \rightarrow \infty} d\left(x_{n}, x_{m}, a, t\right)=0$, that is for each $\varepsilon>0, a \in X$ and for all $t>0$, there exists a natural number $n_{0}$ such that $d\left(x_{n}, x_{m}, a, t\right)<\varepsilon$ for all $n, m \geq n_{0}$.

(3) $(X, d)$ is called complete if every Cauchy sequence in $X$ is a convergent sequence.

Lemma 3. In a parametric 2-metric space, each convergent sequence has at most one limit.

Proof. It is obvious from Definition 3. and Definition 2, therefore omitted.

Remark. In a complete parametric 2-metric space, a convergent sequence need not be a Cauchy sequence. (See [9] for its analogue in 2-metric spaces) 
Example 4. Let $X=\left\{0,1, \frac{1}{2}, \ldots, \frac{1}{n}, \ldots\right\}$ and let the mapping $d: X^{3} \times(0, \infty) \rightarrow \mathbb{R}^{+}$be defined as follows:

$$
d(x, y, z, t)=\left\{\begin{array}{l}
t, \text { if } x, y, z \text { are distinct and }\left\{\frac{1}{n}, \frac{1}{n+1}\right\} \subseteq\{x, y, z\} \text { for some } n \in \mathbb{N} \\
0, \quad \text { otherwise }
\end{array}\right.
$$

Then $(X, d)$ is a complete parametric 2-metric space. Also the sequence $\left(\frac{1}{n}\right)$ is convergent to 0 , but it is not Cauchy.

Definition 6. Let $(X, d)$ be a parametric 2-metric space and $\left(x_{n}\right),\left(y_{n}\right),\left(z_{n}\right)$ be three sequences in $X$ such that $\lim _{n \rightarrow \infty} x_{n}=$ $x, \lim _{n \rightarrow \infty} y_{n}=y$ and $\lim _{n \rightarrow \infty} z_{n}=z . d$ is called continuous if $\lim _{n, m \rightarrow \infty} d\left(x_{n}, y_{m}, z_{n}, t\right)=d(x, y, z, t)$ for all $t>0$. This means that $d$ is called continuous if it is sequentially continuous in all of its three variables.

Remark.If $d$ is a continuous parametric 2-metric on $X$, then every convergent sequence in this space, is a Cauchy sequence.

Definition 7. Let $(X, d)$ be a parametric 2-metric space and $T: X \rightarrow X$ be a self-mapping. Then $T$ is said to be continuous at $x \in X$, if for any sequence $\left(x_{n}\right)$ in $X$ such that $\lim _{n \rightarrow \infty} x_{n}=x$, then $\lim _{n \rightarrow \infty} T x_{n}=T x$. If $T$ is continuous at each point $x$ of $X$, then $T$ is said to be continuous self-mapping on $X$.

Definition 8. A parametric 2-metric space $(X, d)$ is said to be

(1) compact if every sequence in $X$ has a convergent subsequence.

(2) bounded if there exists a real constant $K>0$ such that $d(x, y, z, t) \leq K$ for all $x, y, z \in X$ and for all $t>0$.

Proposition 2. Let $(X, d)$ be a parametric 2-metric space. Then for each $k>0, d_{k}(x, y, z, t)=\min \{d(x, y, z, t), k\}$, for all $x, y, z \in X$ and for all $t>0$, deifnes a bounded parametric 2-metric on $X$ such that the convergence induced by $d$ and the convergence induced by $d_{k}$ are coincident.

Proof. It is enough to show that the tetrahedral inequality (M4) is satisfied, since the other conditions are evident. Let $x, y, z, w \in X$ and $t>0$ be taken arbitrary. Then

$$
\begin{aligned}
d_{k}(x, y, w, t)+d_{k}(x, w, z, t)+d_{k}(w, y, z, t) & =\min \{d(x, y, w, t), k\}+\min \{d(x, w, z, t), k\}+\min \{d(w, y, z, t), k\} \\
& \geq \min \{k, d(x, y, w, t)+d(x, w, z, t)+d(w, y, z, t)\} \\
& \geq \min [k, d(x, y, z, t)\}=d_{k}(x, y, z, t)
\end{aligned}
$$

We see that $\lim _{n \rightarrow \infty} x_{n}=x$ in $(X, d)$, if and only if, for every $a \in X$ and all $t>0, \lim _{n \rightarrow \infty} d\left(x_{n}, x, a, t\right)=0$. This is equivalent that $\lim _{n \rightarrow \infty} d_{k}\left(x_{n}, x, a, t\right)=0$, for all $a \in X$ and all $t>0$. This means that $\lim _{n \rightarrow \infty} x_{n}=x$ in $\left(X, d_{k}\right)$.

Lemma 4. Let $\left(x_{n}\right)$ be a sequence in a parametric 2-metric space $(X, d)$ such that for all $a \in X$ and for all $t>0$,

$$
d\left(x_{n}, x_{n+1}, a, t\right) \leq \alpha d\left(x_{n-1}, x_{n}, a, t\right)
$$

where $0 \leq \alpha<1$ and $n \in \mathbb{N}$. Then $\left(x_{n}\right)$ is a Cauchy sequence in $(X, d)$.

Proof. It is the direct reult of Lemma 1 in [1] and Lemma 2 in [8].

\section{Main results}

In this section, we observe and prove some fixed point results in the parametric 2-metric spaces. While constructing the basement of our observations, we truly keep to the point of the contractive condition " $\rho(T x, T y, a) \leq \ldots$ " observed by 2-metric researchers in their fixed point investigations. We may think of this as meaning that $\rho(x, y, a)$ is a family of distance like functions of $x$ and $y$, indexed by $a \in X$. Since these kinds of contractivity conditions imply colinearity of 
the sequence of iterates starting with any point, it is not easy to find a wide range of applications. Nevetheless, these type of contracive conditions are backbones of the main fixed point results in the literature. Hence, in this section, we study several fixed point theorems based on the parameterized versions of the above contractive conditions.

Theorem 1. Let $(X, d)$ be a complete parametric 2-metric space and $T: X \rightarrow X$ be a continuous self-mapping satisfying the following condition:

$$
d(T x, T y, z, t) \leq \alpha \max \{d(x, y, z, t), d(x, T x, z, t), d(y, T y, z, t), d(x, T y, z, t), d(T x, y, z, t)\}
$$

for all $x, y, z \in X, x \neq y$ and for all $t>0$, where $0 \leq \alpha<1$. Then $T$ has a unique fixed point.

Proof. Let $x_{0} \in X$ be arbitrary and in order to define iterative sequence $\left(x_{n}\right)$ as follows, $T x_{n}=x_{n+1}$ for $n \in \mathbb{N}$. Let write $x=x_{n}$ and $y=x_{n+1}$ in the above inequality, we get

$$
\begin{aligned}
& d\left(T x_{n}, T x_{n+1}, z, t\right) \leq \alpha \max \left\{d\left(x_{n}, x_{n+1}, z, t\right), d\left(x_{n}, T x_{n}, z, t\right), d\left(x_{n+1}, T x_{n+1}, z, t\right), d\left(x_{n}, T x_{n+1}, z, t\right), d\left(T x_{n}, x_{n+1}, z, t\right)\right\} \\
& d\left(x_{n+1}, x_{n+2}, z, t\right) \leq \alpha \max \left\{d\left(x_{n}, x_{n+1}, z, t\right), d\left(x_{n}, x_{n+1}, z, t\right), d\left(x_{n+1}, x_{n+2}, z, t\right), d\left(x_{n}, x_{n+2}, z, t\right), d\left(x_{n+1}, x_{n+1}, z, t\right)\right\} \\
& d\left(x_{n+1}, x_{n+2}, z, t\right) \leq \alpha \max \left\{d\left(x_{n}, x_{n+1}, z, t\right), d\left(x_{n}, x_{n+2}, z, t\right)\right\}
\end{aligned}
$$

Case 1. If $d\left(x_{n+1}, x_{n+2}, z, t\right) \leq \alpha d\left(x_{n}, x_{n+1}, z, t\right)$. By induction we have that $d\left(x_{n+1}, x_{n+2}, z, t\right) \leq \alpha^{n+1} d\left(x_{0}, x_{1}, z, t\right)$, for all $z \in X, t>0$ and $0 \leq \alpha<1$.

From Lemma $4,\left(x_{n}\right)$ is a Cauchy sequence in $X$. Since $(X, d)$ is a complete parametric 2-metric space, $\left(x_{n}\right)$ is convergent to a point $x^{*} \in X$,i.e., $\lim _{n \rightarrow \infty} x_{n}=x^{*}$. Since $T$ is continuous, we obtain that

$$
T x^{*}=T\left(\lim _{n \rightarrow \infty} x_{n}\right)=\lim _{n \rightarrow \infty} T x_{n}=\lim _{n \rightarrow \infty} x_{n+1}=x^{*}
$$

Hence, $T x^{*}=x^{*}$, that is $T$ has a fixed point in $X$.

Case 2. If $d\left(x_{n+1}, x_{n+2}, z, t\right) \leq \alpha d\left(x_{n}, x_{n+2}, z, t\right)$. Then from Lemma 1 in [1], the sequence $\left(x_{n}\right)$ converges to a point $x^{*} \in X$, that is $\lim _{n \rightarrow \infty} x_{n}=x^{*}$. Similar to the Case 1 , the point $x^{*}$ is the fixed point of $T$.

In order to prove the uniqueness let us assume that $T x^{*}=x^{*}$ and $T y^{*}=y^{*}$ where $z \neq x^{*}, z \neq y^{*}$. Then we have

$$
d\left(T x^{*}, T y^{*}, z, t\right) \leq \alpha \max \left\{d\left(x^{*}, y^{*}, z, t\right), d\left(x^{*}, T x^{*}, z, t\right), d\left(y^{*}, T y^{*}, z, t\right), d\left(x^{*}, T y,{ }^{*} z, t\right), d\left(T x^{*}, y^{*}, z, t\right)\right\}
$$

This implies that

$$
d\left(x^{*}, y^{*}, z, t\right) \leq \alpha d\left(x^{*}, y^{*}, z, t\right)
$$

This is true only when $d\left(x^{*}, y^{*}, z, t\right)=0$. Hence $x^{*}=y^{*}$, that is the fixed point of $T$ is unique.

Example 5. Consider the complete parametric 2-metric space $(X, d)$ defined as in Example 4. Define $T: X \rightarrow X$, by $T x=0$, for all $x \in X$. Then Theorem 1 is applicable to $T$.

Corollary 1. Let $(X, d)$ be a complete parametric 2-metric space and $T: X \rightarrow X$ be a continuous self-mapping satisfying the following condition:

$$
d(T x, T y, z, t) \leq \alpha \max \{d(x, y, z, t), d(x, T x, z, t), d(y, T y, z, t)\}
$$

for all $x, y, z \in X, x \neq y, t>0$ and $0 \leq \alpha<1$. Then $T$ has a unique fixed point in $X$. 
Lemma 5. Let $(X, d)$ be a parametric 2-metric space and $T: X \rightarrow X$ be a self-mapping satisfying the following condition: for each $x, y, z \in X$ and for all $t>0$,

$$
d(T x, T y, z, t) \leq \alpha \max \begin{aligned}
& \{d(x, y, z, t), d(x, T x, z, t), d(y, T y, z, t), d(x, T y, z, t), d(y, T x, z, t), \\
& \left.d\left(T^{2} x, x, z, t\right), d\left(T^{2} x, T x, z, t\right), d\left(T^{2} x, y, z, t\right), d\left(T^{2} x, T y, z, t\right)\right\}
\end{aligned}
$$

where $0 \leq \alpha<1$. Then $d\left(T^{2} x, T x, x, t\right)=0$, for all $t>0$. Moreover, we have

$$
d\left(x, T^{2} x, z, t\right) \leq d(x, T x, z, t)+d\left(T x, T^{2} x, z, t\right)
$$

for all $x, y, z \in X$ and for all $t>0$.

Proof. By choosing $z=x$ and $y=T x$ in the above inequality, we obtain

$$
d\left(T x, T^{2} x, x, t\right) \leq \alpha \max \begin{aligned}
& \left\{d(x, T x, x, t), d(x, T x, x, t), d\left(T x, T^{2} x, x, t\right), d\left(x, T^{2} x, x, t\right), d(T x, T x, x, t),\right. \\
& \left.d\left(T^{2} x, x, x, t\right), d\left(T^{2} x, T x, x, t\right), d\left(T^{2} x, T x, x, t\right), d\left(T^{2} x, T^{2} x, x, t\right)\right\}
\end{aligned}
$$

Hence we have that $d\left(T^{2} x, T x, x, t\right) \leq \alpha d\left(T^{2} x, T x, x, t\right)$ for all $x \in X, t>0$. Since $\alpha \in[0,1)$, it is only true when $d\left(T^{2} x, T x, x, t\right)=0$, for all $t>0$.

To prove the second claim let us apply the last inequality of Definition 3,

$$
d\left(x, T^{2} x, z, t\right) \leq d\left(x, T^{2} x, T x, t\right)+d(x, T x, z, t)+d\left(T x, T^{2} x, z, t\right) .
$$

Since $d\left(T^{2} x, T x, x, t\right)=0$, we get $d\left(x, T^{2} x, z, t\right) \leq d(x, T x, z, t)+d\left(T x, T^{2} x, z, t\right)$.

Theorem 2. Let $(X, d)$ be a complete parametric 2-metric space and $T: X \rightarrow X$ be a continuous self-mapping satisfying the following condition:

$$
d(T x, T y, z, t) \leq \alpha \max \begin{aligned}
& \{d(x, y, z, t), d(x, T x, z, t), d(y, T y, z, t), d(x, T y, z, t), d(y, T x, z, t), \\
& \left.d\left(T^{2} x, x, z, t\right), d\left(T^{2} x, T x, z, t\right), d\left(T^{2} x, y, z, t\right), d\left(T^{2} x, T y, z, t\right)\right\}
\end{aligned}
$$

for all $x, y, z \in X, x \neq y$ and for all $t>0$, where $0 \leq \alpha<\frac{1}{2}$. Then $T$ has a unique fixed point.

Proof. Set $x_{0}=x$ and $x_{n}=T x_{n-1}$ for all $n \in \mathbb{N}$. So we have

$$
\begin{aligned}
& d\left(T x_{n-1}, T x_{n}, z, t\right)=d\left(x_{n}, x_{n+1}, z, t\right) \\
& \leq \alpha \max \begin{array}{l}
\left\{d\left(x_{n-1}, x_{n}, z, t\right), d\left(x_{n-1}, T x_{n-1}, z, t\right), d\left(x_{n}, T x_{n}, z, t\right), d\left(x_{n-1}, T x_{n}, z, t\right), d\left(x_{n}, T x_{n-1}, z, t\right),\right. \\
\left.d\left(T^{2} x_{n-1}, x_{n-1}, z, t\right), d\left(T^{2} x_{n-1}, T x_{n-1}, z, t\right), d\left(T^{2} x_{n-1}, x_{n}, z, t\right), d\left(T^{2} x_{n-1}, T x_{n}, z, t\right)\right\}
\end{array} \\
& =\alpha \max \left\{\begin{array}{l}
\left\{d\left(x_{n-1}, x_{n}, z, t\right), d\left(x_{n-1}, x_{n}, z, t\right), d\left(x_{n}, x_{n+1}, z, t\right), d\left(x_{n-1}, x_{n+1}, z, t\right), d\left(x_{n-1}, x_{n+1}, z, t\right),\right. \\
\left.d\left(x_{n}, x_{n+1}, z, t\right), d\left(x_{n}, x_{n+1}, z, t\right)\right\}
\end{array}\right.
\end{aligned}
$$

From Lemma 3,

$$
\begin{gathered}
d\left(x_{n-1}, x_{n+1}, z, t\right)=d\left(x_{n-1}, T T x_{n-1}, z, t\right) \leq d\left(x_{n-1}, T x_{n-1}, z, t\right)+d\left(T x_{n-1}, T T x_{n-1}, z, t\right) \\
d\left(x_{n-1}, x_{n+1}, z, t\right) \leq d\left(x_{n-1}, x_{n}, z, t\right)+d\left(x_{n}, x_{n+1}, z, t\right)
\end{gathered}
$$

Hence we have that

$$
d\left(x_{n}, x_{n+1}, z, t\right) \leq \alpha\left(d\left(x_{n-1}, x_{n}, z, t\right)+d\left(x_{n}, x_{n+1}, z, t\right)\right)
$$




$$
d\left(x_{n}, x_{n+1}, z, t\right) \leq \frac{\alpha}{1-\alpha} d\left(x_{n-1}, x_{n}, z, t\right)
$$

Since $\frac{\alpha}{1-\alpha}<1$, the sequence $\left(x_{n}\right)$ is Cauchy and by the completeness of the space, it converges to some point, say $x^{*} \in X$. By the continuity of the self-mapping, we conclude that $x^{*}$ is the fixed point of $T$, that is $T x^{*}=x^{*}$.

Now let us prove the uniqueness of the fixed point. Assume that $y^{*}$ be another fixed point, that is $T x^{*}=x^{*}$ and $T y^{*}=y^{*}$. So we have

$$
\begin{aligned}
d\left(x^{*}, y^{*}, z, t\right) & =d\left(T x^{*}, T y^{*}, z, t\right) \\
& \leq \alpha \max \begin{aligned}
&\left\{\left(x^{*}, y^{*}, z, t\right), d\left(x^{*}, T x^{*}, z, t\right), d\left(y^{*}, T y^{*}, z, t\right), d\left(x^{*}, T y^{*}, z, t\right), d\left(y^{*}, T x^{*}, z, t\right),\right. \\
& \quad\left.\quad d\left(T^{2} x^{*}, x^{*}, z, t\right), d\left(T^{2} x^{*}, T x^{*}, z, t\right), d\left(T^{2} x^{*}, y^{*}, z, t\right), d\left(T^{2} x^{*}, T y^{*}, z, t\right)\right\}
\end{aligned} \\
& =\alpha d\left(x^{*}, y^{*}, z, t\right) .
\end{aligned}
$$

Therefore, we obtain that $d\left(x^{*}, y^{*}, z, t\right) \leq \alpha d\left(x^{*}, y^{*}, z, t\right)$, for all $z \in X$ and for all $t>0$. This implies that for each $z \in$ $X, \quad d\left(x^{*}, y^{*}, z, t\right)=0$, that is $x^{*}=y^{*}$. Hence we have the uniqueness.

Additionally, $\lim _{n \rightarrow \infty} d\left(T^{n} x, x^{*}, z, t\right)=\lim _{n \rightarrow \infty} d\left(x_{n}, x^{*}, z, t\right)=0$.

This shows that $\lim _{n \rightarrow \infty} T^{n} x=x^{*}$.

Definition 9. Let $(X, d)$ be a parametric 2-metric space and $T: X \rightarrow X$ be a self-mapping. If for all $a \in X$ and all $t>0$, $d\left(T^{n} x, y, a, t\right) \rightarrow 0 \quad(n \rightarrow \infty)$ implies $d\left(T T^{n} x, T y, a\right) \rightarrow 0 \quad(n \rightarrow \infty)$. Then $T$ is said to be an orbitally continuous selfmapping.

Theorem 3. Let $(X, d)$ be a complete bounded parametric 2-metric space and $T: X \rightarrow X$ be an orbitally continuous self-mapping which satisfies the following condition: There exist real numbers $\beta_{1}, \beta_{2}, \beta_{3}, \alpha$ such that $\beta_{1}+\beta_{2}+\beta_{3}>$ $\alpha, \alpha-\beta_{2} \geq 0, \alpha-\beta_{3} \geq 0$ and

$$
\beta_{1} d(T x, T y, z, t)+\beta_{2} d(x, T x, z, t)+\beta_{3} d(y, T y, z, t)-\min \{d(x, T y, z, t), d(y, T x, z, t)\} \leq \alpha d(x, y, z, t)
$$

for all $x, y, z \in X, x \neq y, t>0$ and $0<\alpha<1$. Then for each $x \in X$, the sequence $\left(T^{n} x\right)_{n \in \mathbb{N}}$ converges to a fixed point of $T$.

Proof. Let $x_{0} \in X$ be arbitrary and define $T x_{n}=x_{n+1}$ for $n=0,1,2, \ldots$ If $x_{n}=x_{n+1}$ for all $n$ clearly implies that $\left(x_{n}\right)$ is a Cauchy sequence and the limit of $\left(x_{n}\right)$ is a fixed point of $T$.

Suppose that $x_{n} \neq x_{n+1}$ for each $n=0,1,2, \ldots$ If we write $x=x_{n-1}$ and $y=x_{n}$ in the above inequaility, we have

$$
\begin{gathered}
\beta_{1} d\left(T x_{n-1}, T x_{n}, z, t\right)+\beta_{2} d\left(x_{n-1}, T x_{n-1}, z, t\right)+\beta_{3} d\left(x_{n}, T x_{n}, z, t\right)-\min \left\{d\left(x_{n-1}, T x_{n}, z, t\right), d\left(x_{n}, T x_{n-1}, z, t\right)\right\} \leq \alpha d\left(x_{n-1}, x_{n}, z, t\right) \\
\beta_{1} d\left(x_{n}, x_{n+1}, z, t\right)+\beta_{2} d\left(x_{n-1}, x_{n}, z, t\right)+\beta_{3} d\left(x_{n}, x_{n+1}, z, t\right)-\min \left\{d\left(x_{n-1}, x_{n+1}, z, t\right), d\left(x_{n}, x_{n}, z, t\right)\right\} \leq \alpha d\left(x_{n-1}, x_{n}, z, t\right)
\end{gathered}
$$

So we have

$$
d\left(x_{n}, x_{n+1}, z, t\right) \leq \frac{\alpha-\beta_{2}}{\beta_{1}+\beta_{3}} d\left(x_{n-1}, x_{n}, z, t\right)
$$

If we proceed in this manner we get

$$
d\left(x_{n}, x_{n+1}, z, t\right) \leq \lambda^{n} d\left(x_{0}, x_{1}, z, t\right)
$$

where $\lambda=\frac{\alpha-\beta_{2}}{\beta_{1}+\beta_{3}}<1$. As in the proof of Theorem 1 , we conclude that $\left(x_{n}\right)$ is a Cauchy sequence in the complete parametric 2-metric space $X$. So $\left(x_{n}\right)$ is convergent to some point $x^{*} \in X$. We observe that the limit point $x^{*}$ is the fixed point of $T$. Indeed in this case for all $z \in X$ and all $t>0$, we have $d\left(T^{n} x_{0}, x^{*}, z, t\right) \rightarrow 0$. Since $T$ is orbitally continuous $d\left(T T^{n} x_{0}, T x^{*}, z, t\right) \rightarrow 0$. This implies that $T x^{*}=x^{*}$. 
If $T$ is not orbitally continuous, then we proceed the proof as follows:

$$
\beta_{1} d\left(T x^{*}, T x_{n}, z, t\right)+\beta_{2} d\left(x^{*}, T x^{*}, z, t\right)+\beta_{3} d\left(x_{n}, T x_{n}, z, t\right)-\min \left\{d\left(x^{*}, T x_{n}, z, t\right), d\left(x_{n}, T x^{*}, z, t\right)\right\} \leq \alpha d\left(x^{*}, x_{n}, z, t\right)
$$

Take $n \rightarrow \infty$, we get

$$
\beta_{1} d\left(T x^{*}, x^{*}, z, t\right)+\beta_{2} d\left(x^{*}, T x^{*}, z, t\right) \leq 0
$$

Since $\beta_{1}+\beta_{2}>0$ as $\alpha-\beta_{3} \geq 0$, so $d\left(x^{*}, T x^{*}, z, t\right)=0$ for all $z \in X, t>0$. Hence $T x^{*}=x^{*}$ is the claimed fixed point.

Theorem 4. Let $(X, d)$ be a complete bounded parametric 2-metric space and $T_{1}, T_{2}: X \rightarrow X$ be two self-mappings satisying the following condition:

$$
\beta_{1} d\left(T_{1} x, T_{2} y, z, t\right)+\beta_{2} d\left(x, T_{1} x, z, t\right)+\beta_{3} d\left(y, T_{2} y, z, t\right)-\min \left\{d\left(x, T_{2} y, z, t\right), d\left(y, T_{1} x, z, t\right)\right\} \leq \alpha d(x, y, z, t)
$$

for all $x, y, z \in X$ and for all $t>0$, where $\beta_{1}, \beta_{2}, \beta_{3}, \alpha$ as in Theorem 3. Then $T_{1}$ and $T_{2}$ have a common fixed point.

Proof. Let $x_{0} \in X$ be arbitrary and define the sequence as $\left(x_{n}\right)$ by $x_{2 n+1}=T_{1}\left(x_{2 n}\right), \quad x_{2 n+2}=T_{2}\left(x_{2 n+1}\right)$. Then by the calculations using the inequality given in the hypothesis, we get

$d\left(x_{n}, x_{n+1}, z, t\right) \leq \lambda^{n} d\left(x_{0}, x_{1}, z, t\right)$ with $\lambda=\frac{\alpha-\beta_{2}}{\beta_{1}+\beta_{3}}<1$

Similar to the proof of the above theorems, we conclude that $\left(x_{n}\right)$ is a Cauchy sequence which has some limit say $x^{*} \in X$. Then from the triangle inequality of Definition 2, we get

$$
d\left(T_{1} x^{*}, x^{*}, z, t\right) \leq d\left(T_{1} x^{*}, x^{*}, x_{2 n+2}, t\right)+d\left(T_{1} x^{*}, x_{2 n+2}, z, t\right)+d\left(x_{2 n+2}, x^{*}, z, t\right)
$$

Hence by the assumption, we have

$\beta_{1} d\left(T_{1} x^{*}, x_{2 n+2}, z, t\right)+\beta_{2} d\left(x^{*}, T_{1}^{*} x, z, t\right)+\beta_{3} d\left(x_{2 n+1}, x_{2 n+2}, z, t\right)-\min \left\{d\left(x^{*}, x_{2 n+2}, z, t\right), d\left(x_{2 n+1}, T_{1} x^{*}, z, t\right)\right\} \leq \alpha d\left(x^{*}, x_{2 n+1}, z, t\right)$

which yields by getting $n \rightarrow \infty$,

$$
\left(\beta_{1}+\beta_{2}\right) d\left(T_{1} x^{*}, x^{*}, z, t\right) \leq 0 .
$$

This implies that $d\left(T_{1} x^{*}, x^{*}, z, t\right)=0$, for all $z \in X$ and for all $t>0$. Therefore, $T_{1} x^{*}=x^{*}$.

Similarly, it is seen that $T_{2} x^{*}=x^{*}$. Therefore, $x^{*}$ is the common fixed point of $T_{1}$ and $T_{2}$.

\section{Conclusion}

The notion of metric spaces and its generaliztions with fixed point theory have extensive applications in several branches of mathematics and other disciplines, with various problems in the control theory, convex optimization, decision support systems and so on. Gahler introduced in the 1960's the notion of 2-metric space, and several authors have studied the question of fixed point theorems for mappings on such spaces. If we return to the notion of a parametric 2-metric space, the basic philosophy is that since a parametric 2-metric measures the area according to a parameter, a contraction should send the space towards a configuration of zero area, which is to say a line with respect to a parameter. This result makes hard to find wide real world applications. On the other hand, the application of $b_{2}$-metric spaces to integral equations [15], encouraged us about that our observations may find the right application area. That's why, we intend to study the notion of parametric 2-metric spaces and some fixed points of self-mappings in such spaces. 


\section{Acknowledgement}

I wish to thank to the referees for their constructive suggestions and comments. Also, I would like to express my sincere to Prof.Dr.Halis Aygün who believed and encouraged me during my researches under his supervision.

\section{Competing interests}

The authors declare that they have no competing interests.

\section{Authors' contributions}

All authors have contributed to all parts of the article. All authors read and approved the final manuscript.

\section{References}

[1] An, T. V., Dung, N. V., Hang, V. T. L., General fixed point theorems on metric spaces and 2-metric spaces, Filomat 28 (10), 2037-2045 (2014).

[2] Czerwik, S., Contraction mappings in b-metric space, Acta Math. Inf. Univ. Ostraviensis, 1, 5-11 (1993).

[3] Daheriya, R. D., Shrivastava S., Ughade, M., Parametric metric space, parametric b-metric space and expansive type mappings, Interntional Journal of Mathematics and Its Applications 4(1-C) 107-117, (2016).

[4] Dey, D., Saha, M., Common fixed point theorems in a complete 2-metric space, Acta Univ. Palacki. Olomic., Fac. Ver. Mat., Mathematica 52 (1), 79-87 (2013).

[5] Dhage, B. C., A study of some fixed point theorems. Ph.D. thesis, Marathwada, Aurangabad, India (1984)

[6] Gahler, S., 2-metrische raume und ihre topologische strüktür, Math. Nachr. 26, 115-118 (1963/64).

[7] Huang, L. G, Zhang, X., Cone metric spaces and fixed point theorems of contractive mappings. J. Math. Anal. Appl. 332, 14681476 (2007).

[8] Hussain, N., Khaleghizadeh S., Salimi, P., Abdou, A. A. N., A new approach to fixed point results in triangular intuitionistic fuzzy metric spaces, Abstract and Applied Analysis, vol. 2014, Article ID 690138 (2014).

[9] Hussain, N., Salimi, P., Parvaneh, V., Fixed point results for various contractions in parametric and fuzzy b-metric spaces, Jouranl of Nonlinear Science and Applications, 8, 719-739 (2015).

[10] Khan, M. S., On fixed point theorems in 2-metric space, Publications De L'Insttitut Mathematique, 27(41), 107-112 (1980).

[11] Krishnakumar, R., Sanatammappa, N. P., Fixed point theorems in parametric metric spaces, International Journal of Mathematics Research, 8 (3), 213-220, (2016).

[12] Krishnakumar, R.; Sanatammappa, N. P., Some fixed point theorems in parametric b-metric space. Int. J. Math. Sci. Eng. Appl. 10, 99-106 (2016).

[13] Lahiri, B. K., Das, P., Dey, L. K., Cantor's theorem in 2-metric spaces and its applications to fixed point theorems, Taiwanese Journal of Mathematics, 15(1), 337-352 (2011).

[14] Mustafa, Z, Sims, B., A new approach to generalized metric spaces. J. Nonlinear Convex Anal. 7(2), 289-297 (2006).

[15] Mustafa, Z., Parvaneh, V., Roshan, J. R, Kadelburg, Z., b2-metric spaces and some fixed point theorems, Fixed Point Theory and Applications, 144, 1-23 (2014).

[16] Özgür, E., De La Sen, M., A new perspective on parametric metric spaces, Mathematics, 7, 1-12 (2019).

[17] Priyobarta N., Rohen, Y., Radenovic, S., Fixed point theorems on parametric A-metric spaces, American Journal of Applied Mathematics and Statistics, 6(1), 1-5 (2018).

[18] Sedghi, S.,, Shobe N., Aliouche A., A generalization of fixed point theorems in S-metric spaces, Mat. Vesnik 64(3), 258-266 (2012).

[19] Taş, N., Özgür, N. Y., On parametric S-metric spaces and fixed-point type theorems for expansive mappings, Journal of Mathematics, vol 2016 article $\imath \mathrm{D} 4746732$ (2016).

[20] Taş, N., Özgür, N. Y., Some fixed-point results on parametric $\mathrm{N}_{b}$-metric spaces, Commun. Korean Meth. Soc., preprint. 